\section{TRa 04}

THE GERMAN THOROTRAST STUDY G. Van Kaick

The German Thorotrast Study comprises more than 5.000 persons intravascularly injected with Thorotrast and a similar number of control patients. A total of 2.334 Thorotrast and 1.912 control patients have died, with causes of death established by medical records. The number of death in the Thorotrast versus control series are as follows: Liver cancer $347 / 2$; 1iver cirrhosis 292/42; myeloid leukemia $35 / 3$; chronic Iymphatic leukemia $3 / 2$; bone marrow failure $20 / 1$; bone sarcoma $4 / 1$; plasmocytoma $4 / 1$; lung cancer $46 / 40$; pancreatic carcinoma $20 / 4$; renal carcinoma $4 / 2$. A clear dose effect relationship is present for liver cancer and cirrhosis. How ever, an influence of the bone marrow dose rate to leukemia incidence cannot yet be established. It is one of the most exciting results of the study that there is no excess rate of bronchogenic carcinomas though the lung is exposed to chronic alpha-radiation by the exhaled daughter product Thoron.

Institut für Nuklearmedizin und onkologische Radiologie des Deutschen Krebsforschungszentrums Im Neuenheimer Feld 280, D-6900 Heidelberg 1

\section{RTh 01}

QUALITY ASSURANCE IN COOPERATIVE THERAPY STUDIES (UNDER RADIOTHERAPEUTICAL ASPECTS)

R. Sauer, J. Dunst (a.G.), H.J. Thiel

In cooperative studies, the criteria for selecting patients, the therapeutic regimens and the methods of evaluating are to be defined exactly. Quality assurance programs are an attempt to minimize the protocol deviations and to quantitate the extend of variance. In multimadality studies, a quality assurance program for each modality is required.

As an example of quality assurance in radiotherapy, the experiences of the Ewing $" s$ sarcoma study "CESS 81 " of the German Society for Pediatric Oncology are reported. Following the cancer and Leukemia Group B (CALGB), the quality of radiotherapy can be quantitated in form of a "performance score": the product of the compliance rate and the appropriateness for every participating institution.

Quality assurance programs are essential requirements for comparing the results of institutions in a cooperative group. They control the validity of combined institutional studies, improve the "performance" of the participating institutions and increase the reliability of the collected data.

Quality assurance programs are time consuming, expensive, but basically to reliable statistical analysis of such cooperative studies. A higher scientific quality of these studies can be achieved, if therapeutical results are correlated with the protocol compliance or a "performance score", respectively.

Strahlentherapeutische Klinik und Poliklinik Universität Erlangen-Nürnberg

Universitätsstr. 27, D - 8520 Erlangen

\section{RTh 02}

CISPLATIN, A SENSITIZER OF HYPOXIC MAMMALIAN CELLS?

W. Zlegler

The effect of simultaneous treatment with Cisplatin and gamma Irradiation upon the survival of hypoxic mamalian cells was studied. The four cell lines used were Chinese hamster fibroblasts ( $B$ 14), Syrian hamster kidney cells (HaK), HeLa cells and human melanoma cells (MM). While under aeroblc conditions the presence of Cisplatin had no influence upon the shape of the radiation dose-response curve of $\mathrm{B} 14$ and HeLa cells, in $\mathrm{HaK}$ and MM cells an increased curvature indicated a radiosensitizing effect of Cisplatin. Under hypoxic conditions, Cisplatin did not alter the shape of the radiation survival curve of $B$ 14, HaK and MM cells. In HeLa cells, however, the difference between the curves obtained with radiation only or in the presence of Cisplatin indicates radiosensitization of hypoxic cells down to the survival level of 0.01 , the dose modifying factor roughly equalling 1.2. Combined with irradiation, Cisplatin can act as a specific sensitizer of aerobic cells (e.g. HaK and MM cells) or hypoxic cells (e.g. Hela cells). The drug may also lead to radiosensitization both under aerobic and hypo$x i c$ conditions as in $\mathrm{H} 4$ cells (Int.J.Radiat. Oncol. B101. Phys. I, 929, 1981) or not influence radiosensitivity at all (e.g. B 14 cells). Thus, Cisplatin does not unequivocally act as a hypoxic cell sensitizer in eucaryotic cells. Under the same experimental conditions, Misonidazol showed a marked radiosensitization in all four cell lines, which was specific to hypoxic cells. Strahlenbiologisches Institut der Ludwig-Maximilians-Universität, Schilierstraße 42, D-8000 München, und Abteilung für Strahlenbiologie der GSF, D-8042 Neuherberg. 